\title{
La fortune sourit aux audacieux
}

\author{
Ghislain Bouchard ${ }^{1}$
}

Quelqu'un a récemment provoqué ma réflexion en me lançant un proverbe ou aphorisme qui a fait bien du chemin dans l'imaginaire des philosophes : «La fortune sourit aux audacieux $\gg$.

Joyeuse maxime qui laisse entendre que les audacieux font leur chance, flirtent avec le destin et parviennent à réaliser leurs rêves. Pourtant, la fortune n'est rien, elle n'a pas d'existence, elle est hasard, peut aussi bien faire rire que pleurer, mais si l'on cherche au fond des choses, on s'aperçoit que l'Audacieux entreprend, ce qui explique qu'il réussit. D'autres éléments que la chance paraissent également nécessaires pour arriver à un résultat concluant.

Réflexion, astuce, chance, volonté de faire : autant de leviers pour atteindre cette fortune tant recherchée.

Malheureusement, quand on examine le passé, on voit que la fortune sourit à certaines personnes et pas à d'autres. Elle semble bénir certains projets et pas les autres. Elle aime les gens entreprenants et est comme hypnotisée par celui qui ose. Se sentant incapable de freiner son ardeur, elle va plutôt finir par alimenter, contribuer à engraisser ledit projet.

La Fortune aime la Passion. Elle reconnaît cette qualité chez ceux qu'elle accompagne, soupèse leur ardeur pour détecter les éléments qui motivent et mettent en marche l'Audacieux.

\section{La passion}

La passion, point de départ d'un projet, d'une grande réalisation, parfois même de l'épanouissement d'une vie, est ce faisceau continu d'intérêts qui nous attire vers un objectif. Elle est parfois récompense de l'effort imaginatif. Dans le cas qui nous intéresse, nous essaierons de préciser comment toute notre vie (ma compagne Olivette Hudon et moi) a été vécue à l'enseigne de la passion : passion pour la langue et la possibilité d'illustrer notre pensée par l'expressivité de sentiments tels que l'enthousiasme, la colère, la haine ou l'amour, lesquels mènent infailliblement au désir de développer les instruments par excellence que sont la maîtrise de la langue parlée et la capacité d'enrichir son emploi de manière efficace, voire dramatique. Dans un deuxième temps, nous nous sommes attaqués à l'art de communiquer à notre entourage les arcanes de la visualisation et de l'animation.

\section{La séduction}

La fortune sourit aux audacieux qui n'hésitent pas à faire examen de tout ce qui bouge autour d'eux pour y détecter d'éventuels acteurs susceptibles de rejoindre l'Audacieux dont il est question. Cet Astucieux ne manque pas de faire miroiter les couleurs de sa propre passion et la possibilité que les participants développent une certaine envie de séduction auprès $\mathrm{du}$ public tout autour d'eux-mêmes. En somme, il faut séduire pour inciter à ce que ce phénomène se multiplie auprès d'autres participants.

\section{L'équipe}

L'Audacieux s'est fixé un but qu'il va expliciter, développer, amplifier avec le temps. Il commence donc par convertir des fidèles à sa cause, à les exciter face au but à atteindre : une communication la plus parfaite possible avec des publics par le biais du théâtre. Petit à petit se dégagent, chez les élus, des aptitudes, des goûts personnels qui leur permettent de s'impliquer plus aisément dans les différentes facettes du spectacle. Chose étonnante, quand on se joint à un groupe orienté vers la création, on se réveille quelquefois 
dans les bottines d'un personnage qu'on n'avait pas imaginé. Combien de volontaires se retrouvent au maquillage, à la fabrication des décors ou aux accessoires alors que, dans leur for intérieur, ils rêvaient de personnifier la jeune héroïne ou de camper le jeune premier. Mais l'Audacieux veille au grain et il ne manque pas de faire partager sa vision globale: le bien collectif. De toute manière, une fois intégré dans le groupe, le postulant devient porteur du projet sans savoir ce que lui réserve le prochain spectacle. J'en connais qui furent balayeur de scène avant de créer des décors ou souffleuse avant d'attraper des rôles plus spectaculaires.

\section{La faisabilité}

La fortune sourit à l'Audacieux qui garde un pied sur terre. Tout le monde comprendra qu'il a à faire face à des problèmes purement mercantiles, voire à des montages financiers. Bien sûr, l'honnêteté de l'opération ne doit jamais être mise en doute. La fortune sourit parfois aux audacieux tricheurs, pour qui tous les moyens sont bons. Il va de soi que, dans l'univers des Arts, et particulièrement du théâtre, dans lequel nous avons œuvré toute notre vie, les sommes impliquées sont rarement astronomiques. Il y a peu de danger que l'Audacieux se transforme en exploiteur. Le théâtre, surtout amateur, demeure une aventure risquée, même pour un Audacieux prudent.

\section{La bonne affaire}

Néanmoins, l'Audacieux doit flairer la bonne affaire, sauter sur l'occasion, convaincre, entreprendre, poursuivre sa passion jusqu'au succès. Ce qu'on a dit du théâtre amateur peut s'appliquer à bien d'autres activités artistiques; même là, parmi la centaine de spectacles que nous avons réalisés, plusieurs avaient une certaine prétention au professionnalisme et impliquaient des budgets de cette dimension.

\section{Le théâtre, surtout amateur, demeure une aventure risquée, même pour un Audacieux prudent.}

\section{Un peu d'histoire}

Essayons maintenant d'appréhender le monde concret que nous avons connu et parfois bouleversé, mais également fait rigoler au cours des 50 dernières années.

Un monde aux activités culturelles encore timides mais qui allait connaître une éclosion pendant la première moitié du siècle et son apogée dans la seconde moitié, en incluant les grands spectacles historiques.

Des années 1930 jusqu'au milieu du siècle, deux foyers sont particulièrement vivaces : à Chicoutimi, la Société des concerts et l'activité théâtrale au Séminaire se développèrent année après année; à Hébertville, il existait une société constituée de professionnels de différentes catégories, lesquels montèrent et jouèrent, pendant plusieurs années, la pièce du Père Laurent Tremblay: "Le Curé Hébert ». Ce même Laurent Tremblay fut également à l'origine des nombreux pageants montés à l'époque. Celui de 1938 était encore dans les mémoires lorsqu'on a commencé à écrire «La Fabuleuse ». Il fut d'ailleurs présenté sur des collines de la Grande-Baie. Puis vinrent les pageants de Chicoutimi, en 1942, de Jonquière, en 1947 et d'Hébertville, en 1949. Il s'agissait déjà d'un accomplissement inspiré des modèles européens qui dépassait toute attente. Le spectacle dit « du Centenaire » de 1938 réunissait même au-delà de 1000 participants ${ }^{2}$.

La fin du siècle allait ramener ces grandes manifestations-spectacles à l'avant-scène. La première et plus percutante fut évidemment « La Fabuleuse Histoire d'un Royaume » qui, en 17 ans, a connu au-delà de 400 représentations devant des publics se chiffrant à plus de 875000 spectateurs. Personne ne prévoit la fin de sa «vie utile». D'autres productions furent orientées vers les mêmes objectifs: "Le Tour du Monde de Jos Maquillon », ou «L'Épopée des Patriotes », fut joué en 1994, 1995 et 1997. L'histoire du village de Laterrière fut célébrée sous le titre de «La Tournée Folle du Grand-Brûlé » et soulignait les 150 années écoulées depuis l'établissement d'une coopérative de développement agricole. En 1997, à Desbiens, site du poste de traite de Métabetchouan, un nouveau spectacle historique à grand déploiement célébra le $350^{\mathrm{e}}$ anniversaire de la découverte du Piékouagami par le Père Jean Dequen, qui le baptisa « Lac Saint-Jean ».

Ces quatre grands spectacles historiques furent montés essentiellement avec les mêmes équipes de création, c'est-à-dire Dominique et Jean-François Laprise 
pour la musique, Olivette Hudon pour les costumes, Bastien Bouchard pour le support technique, Stéphanie Bouchard pour la mise en scène et Ghislain Bouchard comme auteur et metteur en scène.

À la même époque, soit 1997, une équipe très considérable de Jonquiérois écrivit et présenta, en grande pompe, "Le Rêve de Marguerite Belley », d'Yvon Paré et Danielle Dubé. Malheureusement, malgré ses qualités certaines, le spectacle ne fit pas ses frais et ne fut pas repris.

Voilà, dans ses grandes lignes, l'histoire de ces productions historiques à grande dimension qui étayèrent le $20^{\mathrm{e}}$ siècle chez nous. Par ailleurs, l'expérience que nous avions acquise au Séminaire aussi bien qu'à l'Université fut mise à profit lors de la création, par Olivette et moi, de diverses troupes régionales :

- «Le Théâtre du Coteau », à Chicoutimi, de 1955 à 1961;

- « La Marmite », à Jonquière, de 1962 à 1969;

- « Les Pédagos », à l'École normale Cardinal-Bégin d'Arvida, de 1963 à 1969;

- «Les Vaincus », au Centre culturel de Jonquière, de 1971 à 1973;

- «Théâtruc », de 1969 à 1977, à l'Université du Québec à Chicoutimi.

Ces troupes permirent de créer près d'une centaine de spectacles et furent à l'origine d'un mouvement théâtral intense dans la région. Il suffit de citer la fondation du théâtre populaire d'Alma (TPA), dont faisait partie le bouillant Jacques Brassard qui connut, par la suite, une carrière politique impressionnante; le « Mic-Mac », à Roberval, créé dans les années 1970 et encore actif. J'ai été heureux d'aider à la mise en scène de leur premier spectacle : «Du vent dans les branches de sassafras ».

Ces troupes permirent de créer près d'une centaine de spectacles et furent à l'origine d'un mouvement théâtral intense dans la région.

\section{La foire culturelle}

L'événement marquant des années 1970 fut la création de la «Foire culturelle », à l'Université du Québec à Chicoutimi. Elle a été, pendant six ou sept ans, lieu de rencontre, de recherche, d'expositions multiples $^{3}$ telles que celles de Johann Krieber, Charles Daudelin et Denys Tremblay, de création de dizaines de pièces de théâtre, dans ce petit théâtre invraisemblable du $5^{\mathrm{e}}$ étage du premier pied-à-terre de l'Université du Québec à Chicoutimi.

Évidemment, la plupart de ces troupes venaient de la région et je crois sincèrement que cette occasion de jouer a été profitable à plusieurs compagnies. C'est là, entre autres, que se fit la première vraie sortie des «Échassiers » de La Baie, lesquels donneraient naissance, plus tard, à rien de moins que «Le Cirque du Soleil ».

\section{L'événement marquant des années 1970 fut la création de la « Foire culturelle », à I'Université du Québec à Chicoutimi.}

\section{L'opérette du Carnaval-Souvenir}

Le Carnaval-Souvenir fut propice à de nombreuses expériences de scène. De "Grand-maman reçoit » à "Forges et Dentelles », ou au «Grand-Feu », puis à «l'Opérette», ces spectacles furent occasion de manifester leur talent à des centaines de comédiens, danseurs, chanteurs, musiciens, créateurs de costumes et de décors. En ce qui nous concerne, Dominique, Jean-François Laprise et moi, le Carnaval fut l'occasion de créer notre première grande comédie musicale «Entre Deux-Temps ». Cette pièce nous a permis de vérifier bon nombre d'éléments qui devaient nous permettre de donner naissance à ces grands spectacles qui meubleront la fin du siècle: " La Fabuleuse Histoire d'un Royaume », "Le Tour du Monde de Jos Maquillon », «La Tournée Folle du Grand-Brûlé » et « La Grande Embardée ».

\section{Conclusion}

En conclusion, il est important de souligner les très nombreuses initiatives qui se sont multipliées partout dans la région depuis une quinzaine d'années. De Dolbeau (Madiluth) à Arvida (Québecissime), à Jon- 
quière, (La Rubrique, le Côté Cour, l'Atelier d'Opéra), à Chicoutimi (Ecce Mundo), à l'Université du Québec à Chicoutimi, (Les Têtes Heureuses), à Métabetchouan (le Camp Musical), à Saint-Bruno (le Prisme Culturel), à La Baie (le Bal des Lasers), un nombre presque incalculable de productions, dont certaines feront le tour du Québec et de la France, entre autres «La Fabuleuse Histoire d'un Royaume » présentée à deux reprises devant 20000 spectateurs en Normandie (1990 et 1992), et «Québecissime » qui fit la même traversée à quelques reprises. Dieu sait quand la tempête s'arrêtera.

\section{Il est important de souligner les très nombreuses initiatives qui se sont multipliées partout dans la région depuis une quinzaine d'années.}

\section{Notes et références}

1 L'auteur est historien de formation, passionné de l'histoire et du théâtre, et amant inconditionnel de la nature.

2 Voir l'analyse extrêmement détaillée qu'en fait l'auteur Rémi Tourangeau dans son ouvrage : «Fêtes et Spectacles du Québec (Saguenay-Lac-Saint-Jean) ». L'ouvrage de recherche post doctoral de 400 pages présente une introduction intitulée: "Culture et identité au Saguenay-LacSaint-Jean » de même qu'une analyse comparative du pageant du Centenaire et de «La Fabuleuse Histoire d'un Royaume », ainsi que le livret des deux spectacles.

3 Qui pourrait oublier les 250 photos géantes de Johann Krieber sur le thème de l'environnement, un artiste dont la réputation s'étendait d'un océan à l'autre; ou l'invraisemblable environnement créé par le sculpteur Charles Daudelin, parmi les plus grands maîtres au Québec; ou les fantaisies surprenantes de Denys Tremblay, ex-roi de l'Anse? 